\title{
Cavity-induced backscattering in a two-dimensional photonic topological system
}

\author{
Yuhao Kang $\odot$ and Azriel Z. Genack ${ }^{*}$ \\ Department of Physics, Queens College and Graduate Center of the City University of New York, Flushing, New York 11367, USA
}

(Received 23 October 2019; revised manuscript received 15 January 2020; accepted 4 February 2020; published 27 February 2020)

\begin{abstract}
Topological protection of transmission has been demonstrated in the edge channel in topological insulators in the face of bent paths and on-site randomness in the structure. Microwave measurements and couple-mode theory of a topological medium possessing time-reversal symmetry with a cavity adjacent to the edge channel show that spin is not conserved in scattering from the defect. The transmission time is negative near resonance with the defect.
\end{abstract}

DOI: 10.1103/PhysRevResearch.2.013221

\section{INTRODUCTION}

Recent progress in the study of photonic topological structures has opened new possibilities for robust wave transport from microwave to optical frequencies [1,2]. Unimpeded transport through edge states has been demonstrated along paths on the boundary between topological and trivial domains with different topological invariants when the path is bent and when it is surrounded by distributed defects [3].

Several structures have been proposed to emulate the quantum spin Hall (QSH) effect and test the robustness of edge modes. Hafezi et al. [4-6] proposed a network of coupled optical ring resonator waveguides in which the pseudospin corresponds to clockwise and counterclockwise circulation in the ring. They showed in optical experiments and numerical simulations that transmission through the edge mode remains near unity in the face of random fluctuations in the frequencies of ring resonators comprising the system as the system size increases. QSH-like topological insulators (TIs) were also realized in bianisotropic metamaterials [7,8]. The measured transmission time of edge modes averaged over disorder is consistent with ballistic propagation [9].

In addition to perfect transmission, the ability to control the transmission time in paths within the TI could be important in many applications such as optical buffer and signal processing [10]. The time delay can be modified by creating localized modes inside the bulk region [11-13] by introducing disorder, by changing the length of the boundary line between domains by introducing bends in the boundary line, or by gradually changing the lattice parameter away from the edge [14]. The impact of defects is also of interest because of inevitable disorder introduced in the fabrication of a topological structure, which produces discrete modes in

\footnotetext{
*genack@qc.edu

Published by the American Physical Society under the terms of the Creative Commons Attribution 4.0 International license. Further distribution of this work must maintain attribution to the author(s) and the published article's title, journal citation, and DOI.
}

the vicinity of the edge. Several different types of disorder have been considered, including highly correlated disordered crystals [15] and random fluctuations in on-site energy [16]. An immediate question is whether unidirectional propagation of the edge mode is maintained when a defect cavity covering several lattice sites and supporting several modes within the bulk band gap is introduced near the domain edge. This is of particular interest because it is possible to engineer the defect and its proximity to the edge to slow down the edge mode by coupling to such states at particular frequencies.

The magnitude and phase of the field transmitted through a continuum channel coupled to a discrete mode have the characteristics of a Fano resonance. The Fano resonance was first proposed to explain the asymmetric spectrum of the electron-molecule scattering cross section [17] arising from the interference between the freely propagating continuum of electron states and the discrete autoionized state. This phenomenon can be extended to classical interference between a discrete state with narrow linewidth and a broad continuum state. The sharp dip in transmission in a Fano resonance has many applications in photonic crystal waveguide structures such as an optical switch from complete transmission to complete reflection [18]. Here we observe a Fano resonance in a nontrivial photonic crystal between the edge mode and several discrete modes of an adjacent cavity within the band gap. We will analyze the observed spectrum using coupled-mode theory. Understanding the coupling between the edge mode and modes of a cavity is the first step in tailoring transport in TIs via cavity modes $[6,19]$.

We study a TI with time reversal (TR) symmetry. This system does not require real or auxiliary magnetic fields to realize topological protection in bent paths and weak on-site distributed disorder. The sample has a topological domain with the triangular lattice shown in the upper half of Fig. 1(a) adjacent to a trivial domain with the triangular lattice shown in the lower half of the figure. The topological lattice is composed of rods with a concentric collar. The position of the collar between two bounding plates can be changed by pushing the rod which protrudes through holes of a diameter of $0.318 \mathrm{~cm}$ in the plates, which are separated by $1.089 \mathrm{~cm}$. 
(a)

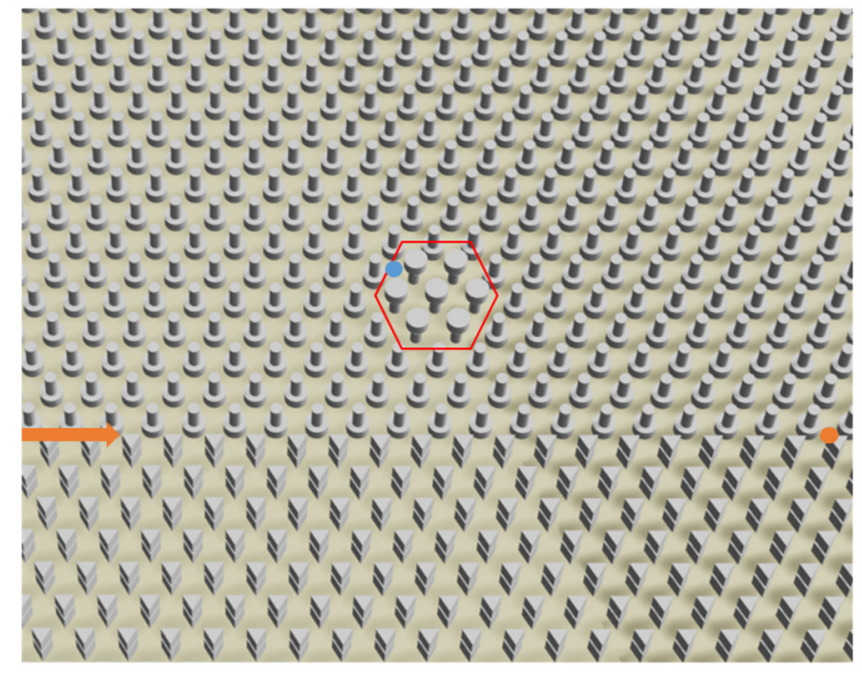

(b)

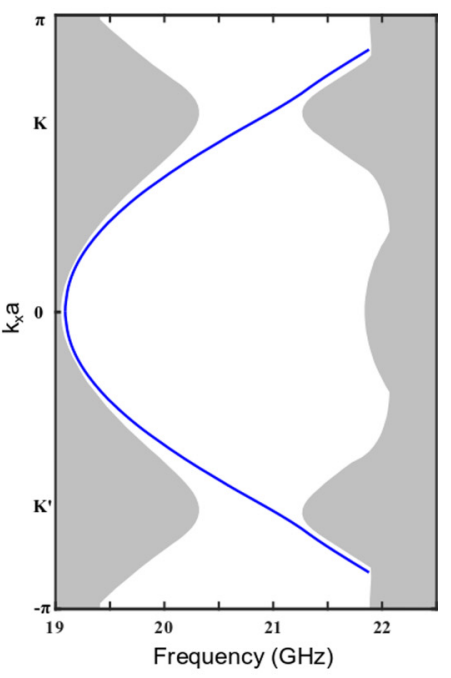

(c)

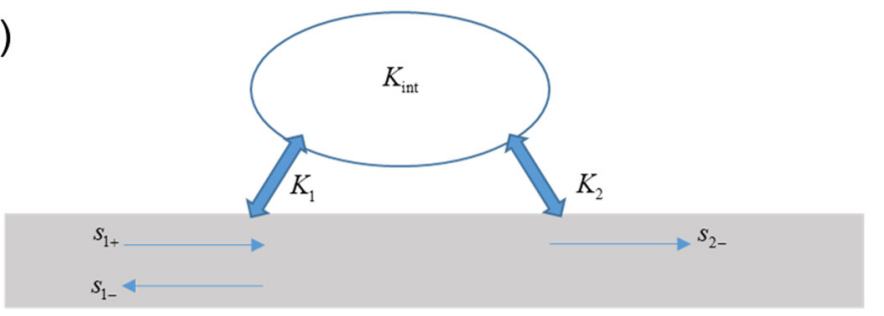

FIG. 1. Schematic diagram of the structure. (a) Structure without the upper plate. The red arrow indicates the direction of flow of the incident wave. The blue and orange points are the positions of two detectors located within the cavity and at the output of the sample, respectively. The sample parameters are given in Ref. [7]. They are as follows: The overall dimensions of the sample are $30 \times 40 \mathrm{~cm}^{2}$. Lattice constant: $1.0890 \mathrm{~cm}$; spacing between plates: $1.0890 \mathrm{~cm}$; height of collar: $0.3580 \mathrm{~cm}$; diameter of collar: $0.6215 \mathrm{~cm}$; diameter of rod: $0.3175 \mathrm{~cm}$; height of triangular prism: $0.5040 \mathrm{~cm}$; gap between triangular prisms: $0.0810 \mathrm{~cm}$; side length of triangular prism: $0.5020 \mathrm{~cm}$. Within the red border, rods are pushed up so that the attached collars are in contact with the upper plate. The real structure contains 15 layers of triangular prisms and 25 layers of rods. (b) Band structure of edge mode. The bulk band gap extends from 20.3 to $21.3 \mathrm{GHz}$. (c) Schematic of coupled-resonator model. $K_{1} / K_{2}$ is the coupling coefficient between the cavity mode and the left or right side of the edge channel. $K_{\text {int }}$ denotes the internal loss of the cavity.

The crystal elements and the top and bottom plates are made of low-resistivity copper. In the experiment, the collars on the rods are always pushed to touch one of the plates. To create a cavity, seven rod-collar units are pushed to be in contact with the upper plate. A second set of holes with diameters of $1 \mathrm{~mm}$ allows the source and detector antenna to be inserted into the metacrystal. The entire length of the dipole antenna of approximately $7 \mathrm{~mm}$ is inserted into the sample.

The waveguide supports both transverse-electric-like (TE) and transverse-magnetic-like (TM) modes. When the collar is midway between the plates, the TE and TM modes are degenerate at the Dirac point. Pseudo-spin-up and pseudo-spindown states are the bonding and antibonding combinations of these modes. TE and TM modes are coupled when the collar is displaced from the midpoint between the plates, effectively emulating spin-orbit coupling in electronic systems and leading to topological order. This system can be described by the Kane-Mele model $H=v\left(\delta k_{x} \widehat{\tau_{z}} \widehat{s_{0}} \widehat{\sigma_{x}}+\delta k_{y} \widehat{\widehat{C}_{0}} \widehat{s_{0}} \widehat{\sigma_{y}}\right)+m \widehat{\tau_{z}} \widehat{s_{z}} \widehat{z_{z}}$, where the Pauli matrices $\widehat{\tau}, \widehat{s}$, and $\widehat{\sigma}$ act on the subspaces of valley, spin, and double states, respectively. $v$ is the group velocity, and $m$ is the mass term due to bianisotropy [7]. This Hamiltonian is a good approximation near the Dirac point.
Pushing the rod to the opposite plate changes the sign of $m$. The electromagnetic wave is confined to the boundary between the two-dimensional (2D) lattice of rods and collars and the 2D array of triangular prisms with a gap between them, which form a trivial insulator with a band gap that coincides with that of the adjacent TI [7,20]. Two pseudospin-polarized edge modes propagate in opposite directions along the interface to form a Kramers pair.

In an electronic TI with TR symmetry, the Kramers theorem ensures the decoupling of a single pair of helical edge states [21]. Previous theoretical, numerical, and experimental studies show that spin flipping in a photonic system is inhibited, and reflection is suppressed. Although the effect of point defects and bent path have been discussed, there has not been much experimental study of the effect of a large cavity near the edge channel on the reflection rate.

In this work, we measure microwave propagation in a QSH TI in which a single defect cavity is introduced at different distances from the edge. We extract the reflection through a fit of an analytical model of the Fano resonance of the continuum edge mode and cavity modes to measurements of field spectra inside the cavity. 
TABLE I. The complex central frequency $\omega_{n}-i \Gamma_{n \text { tot }}$ of modes extracted from measurement (GHz).

\begin{tabular}{|c|c|c|c|c|c|}
\hline & \multirow[b]{2}{*}{ Numeric eigenmode } & \multicolumn{4}{|c|}{ Measurement (GHz) } \\
\hline & & $a$ & $b$ & $c$ & $d$ \\
\hline 1 & 20.376 & $20.397-0.014 i$ & $20.397-0.039 i$ & $20.364-0.0237 i$ & $20.376-0.0054 i$ \\
\hline 2 & 20.502 & $20.464-0.0268 i$ & $20.478-0.0251 i$ & $20.488-0.0247 i$ & $20.481-0.0253 i$ \\
\hline 3 & 20.571 & $20.707-0.0187 i$ & $20.616-0.0305 i$ & $20.716-0.0336 i$ & $20.587-0.0199 i$ \\
\hline 4 & 20.572 & $20.812-0.0217 i$ & $20.812-0.0243 i$ & $20.811-0.0262 i$ & \\
\hline 5 & 21.043 & $20.922-0.028 i$ & $20.931-0.0248 i$ & $20.932-0.0284 i$ & $20.939-0.0198 i$ \\
\hline 6 & 21.046 & $21.046-0.0207 i$ & $21.053-0.0164 i$ & $21.027-0.0488 i$ & $21.052-0.0184 i$ \\
\hline
\end{tabular}

\section{COUPLED-MODE THEORY}

To analyze the measured tramssion spectrum and relate it to the narrow-bandwidth modes inside the system, we apply the coupled-mode theory. This model treats the propagation of waves in a sample with a continuum channel and multiple modes and internal loss in the cavity [22,23]. The complex field of the $n$th mode $a_{n}$ with central frequency $\omega_{n}, a_{n} \propto e^{-i \omega t}$, evolves as

$$
\begin{aligned}
\frac{d a_{n}}{d t} & =\left(-i \omega_{n}-\Gamma_{n 1}-\Gamma_{n 2}-\Gamma_{n \text { int }}\right) a_{n}+K^{T}\left|s_{+}\right\rangle, \\
\left|s_{-}\right\rangle & =C\left|s_{+}\right\rangle+D a .
\end{aligned}
$$

Here $C=\left[\begin{array}{cc}r & t^{\prime} \\ t & r^{\prime}\end{array}\right]$ is the scattering matrix directly through the continuum state, $a=\left(a_{1}, a_{2} \cdots a_{n}\right)^{\tau} ; \Gamma_{n 1}, \Gamma_{n 2}, \Gamma_{n i n t}$ denote the decay rates of the $n$th cavity mode due to coupling to the left and right and internal loss, respectively. $K$ and $D$ are the coupling matrices between discrete modes and the incoming and outgoing ports, respectively. $K=$ $\left[\begin{array}{cccc}K_{11} & K_{21} & \cdots & K_{n 1} \\ K_{12} & K_{22} & \cdots & K_{n 2}\end{array}\right] ; K_{n 1 / 2}$ is the strength of coupling between the $n$th cavity and port $1 / 2 .\left|K_{n 1 / 2}\right|^{2}=2 \Gamma_{n 1 / 2}$ [23]. Due to electromagnetic reciprocity, $D=K$ [24]. $\left|s_{ \pm}\right\rangle=\left(s_{1 \pm}, s_{2 \pm}\right)^{\tau}$, with $s_{2+}=0$. From Eq. (1), we find $a_{n}=\frac{K_{n 1} s_{1+}}{i\left(\omega_{n}-\omega\right)+\Gamma_{n \text { tot }}}, s_{2-}=$ $t s_{1+}+\sum_{n} K_{n 2} a_{n}$. The transmission coefficient can then be expressed as

$$
\tau=\frac{s_{2-}}{s_{1+}}=t+\sum_{n} \frac{K_{n 1} K_{n 2}}{i\left(\omega_{n}-\omega\right)+\Gamma_{n \text { tot }}},
$$

where $\Gamma_{n \text { tot }}=\Gamma_{n 1}+\Gamma_{n 2}+\Gamma_{n \text { int }}$ is the total linewidth of the $n$th mode.

\section{EXPERIMENTAL RESULTS AND SPECTRAL ANALYSIS}

To determine the linewidth and central frequency of the cavity modes, we measure the spectrum of the field inside the cavity. The source is far from the cavity. Only spin-uppolarized light can propagate along the edge and interact with the cavity mode. The spin-down component decays evanescently along the edge. The probe is placed at the upper-left corner of the cavity, which is indicated by the blue point in Fig. 1(a). The position of the probe relative to the cavity is fixed in measurements in which the separation of the cavity from the boundary line is changed. The results for separations between the cavity and boundary of one, three, five, and seven layers are shown in Fig. 2. The red curves in the upper panels of Figs. 2(a)-2(d) are the measured signals. The blue dotted curves are the fits to the data based on the superposition of the $a_{n}$ obtained from Eq. (1):

$$
E(\omega)=\sum_{n} \frac{V_{n}}{\omega-\omega_{n}+i \Gamma_{n \text { tot }}} .
$$

Here $V_{n}$ describes the coupling between the modes of the cavity and the edge mode, which depends on the detector position and field distributions in the cavity modes.

The parameters $\omega_{n}$ and $\Gamma_{n \text { tot }}$ are obtained from the modal decomposition of the spectrum using the method of harmonic inversion [25]. The blue dotted curves in the upper panels of Figs. 2(a)-2(d) show the fit to the red curves of the measured spectra. The lower panels in these figures show the contribution of the individual modes between 19.5 and $22.5 \mathrm{GHz}$. The coupling strength decreases when the cavity is moved further from the edge. Table I lists the complex central frequency $\omega_{n}-i \Gamma_{n \text { tot }}$ extracted from the measurements in the range from 20.3 to $21.3 \mathrm{GHz}$. The absolute value of the imaginary part of the frequency is the half linewidth of the mode. The first column gives the eigenfrequencies of isolated cavity modes found using COMSOL MODE SOLVER. Intensity patterns of these eigenmodes are shown in Fig. 2(e).

The first, second, fifth, and sixth eigenmodes are observed in measurements. No mode is found in experiments that are centered near the frequencies of the third or fourth modes of an isolated cavity. We also measured spectra at other positions within the defect but did not observe an evident signal at the frequency of the third or fourth cavity mode. These two modes are more extended spatially than the others and overlap more strongly with the edge. These modes may therefore be shifted and broadened more than the other modes and they may correspond to other peaks. They may not be seen in Fig. 2(d) because the signal is smaller than in Figs. 2(a)-2(c).

Spectra of the intensity transmission coefficient and the phase shift are shown in Figs. 3(a) and 3(b). An abrupt phase change of $-0.6 \pi \mathrm{rad}$ is observed at $21.06 \mathrm{GHz}$. The intensity is near zero at this point, as expected for a Fano resonance. The mode analysis described above shows that the transmission spectrum is the result of the interference between the edge mode and the cavity mode at 20.922-0.02 $8 i \mathrm{GHz}$. The small neighboring peaks are due to nearby modes.

We first consider the case of a single localized mode and simplify Eq. (2) to

$$
\tau=t+\frac{2 \sqrt{\Gamma_{1} \Gamma_{2}}}{i\left(\omega_{0}-\omega\right)+\Gamma_{\text {tot }}} e^{i \varphi} .
$$


(a)

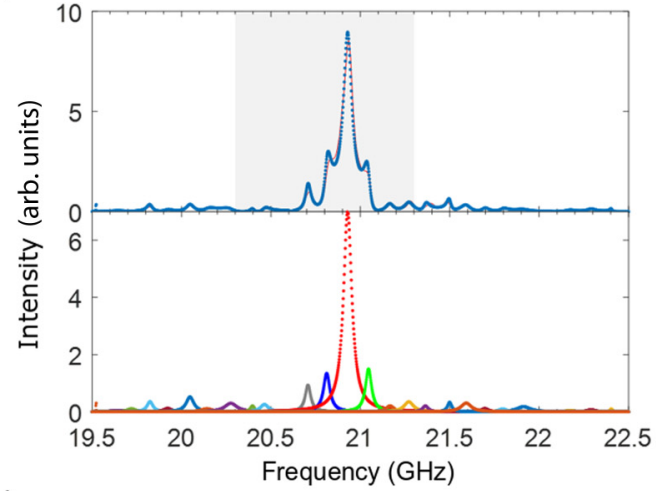

(c)

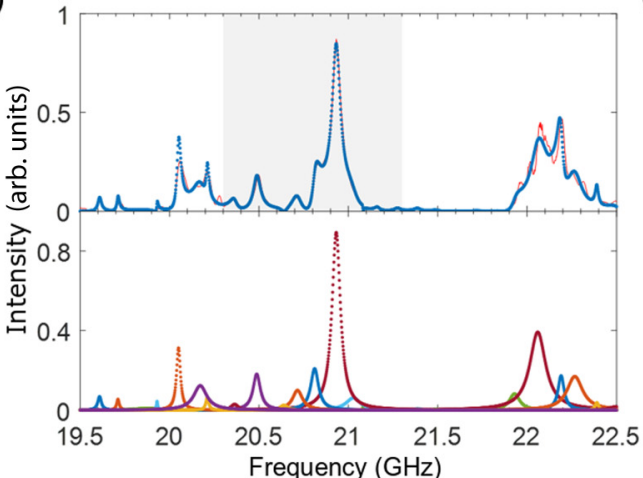

(b)

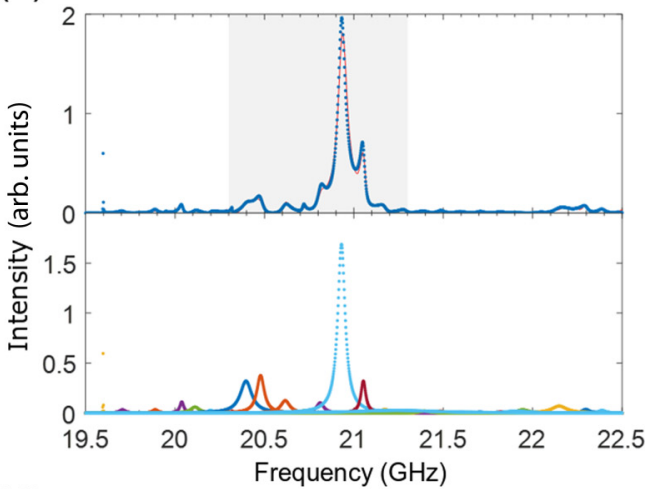

(d)

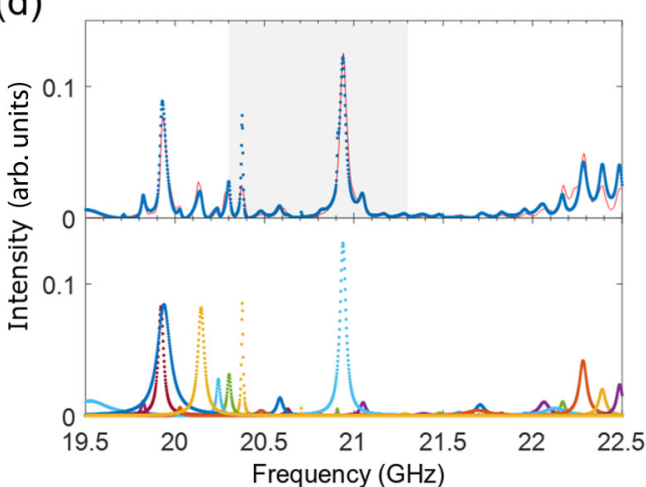

(e)
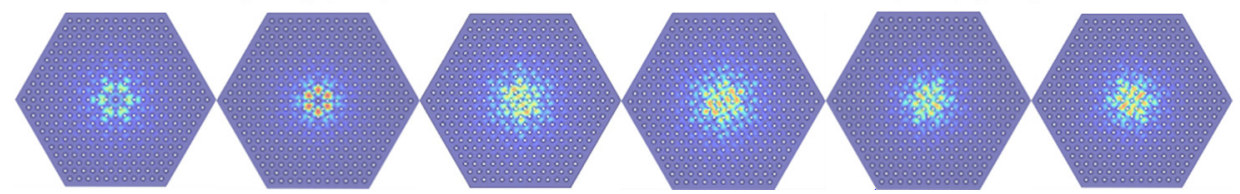

FIG. 2. Measurement of cavity modes. (a)-(d) Upper panel: The red curve is the measured spectrum; the blue dotted curve is the fit to the data based on the harmonic inversion method. The band gap is indicated by the shaded region. Lower panel: The contribution of each of the quasinormal modes. The distances between the cavity and the boundary line are one, three, five, and seven layers in (a)-(d), respectively. (e) Numerical calculation of the cavity eigenmodes within the bulk of the sample isolated from the edge. From left to right, the modes correspond to the six numeric eigenmodes in Table I.

The subscript $n$ is omitted and $\varphi$ is the phase difference between the resonant term and the direct coupling term $t$, which can be taken to be a constant real number in the narrow frequency range near the resonance
Since the scattering matrix is not unitary in a dissipative system, another constraint is needed to determine $\varphi$. Yoon et al. treat the quasireversibility [26] of the scattering matrix as follows: In a dissipative system, $\left|s_{-}\right\rangle=S_{\text {loss }}\left|s_{+}\right\rangle$. From Eq. (1) (a)

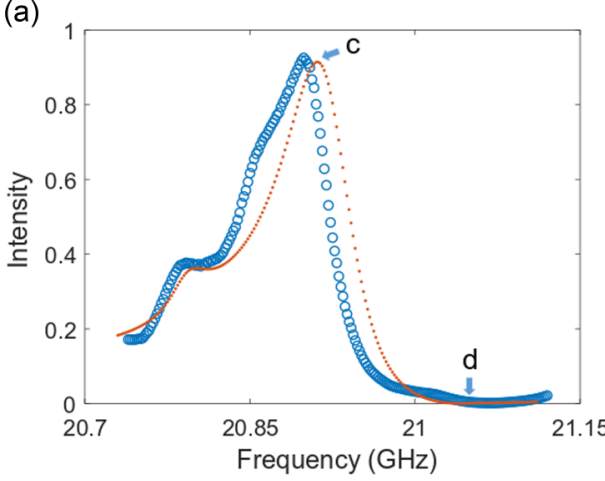

(b)

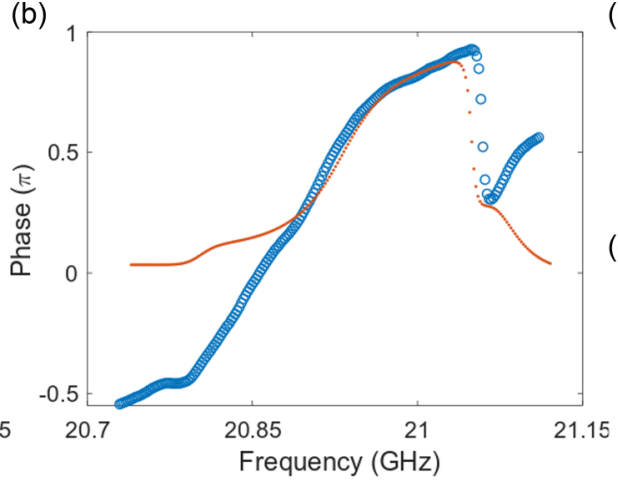

(c)

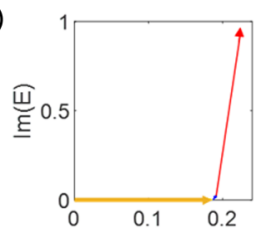

(d)

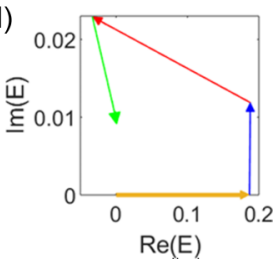

FIG. 3. Transmission spectra. Spectra of (a) transmission coefficient and (b) phase at the edge on the output surface. The blue and red curves indicate the measured and analytical results, respectively. (c), (d) Representations in the complex plane of individual modal contributions at the frequencies indicated by arrows $c$ and $d$ in (a). The orange vector indicates the edge channel, while the blue, red, and green vectors correspond to the modes represented by the blue, red, and green dashed lines at around $21 \mathrm{GHz}$ in the lower panel of Fig. 2(a). 
we obtain $S_{\text {loss }}=C+\frac{K K^{T}}{i\left(\omega_{0}-\omega\right)+\Gamma_{1}+\Gamma_{2}+\Gamma_{\text {int }}}$. In the case of time reversal, $\left|s_{+}\right\rangle^{*}$ will be scattered to $\left|s_{-}\right\rangle^{*}$, while the internal loss is replaced by gain, so that $\left|s_{+}\right\rangle^{*}=S_{\text {gain }}\left|s_{-}\right\rangle^{*}$. By replacing $\Gamma_{\text {int }}$ with $-\Gamma_{\text {int }}$, we obtain $S_{\text {gain }}=C+\frac{K K^{T}}{i\left(\omega_{0}-\omega\right)+\Gamma_{1}+\Gamma_{2}-\Gamma_{\text {int }}}$. Since $S_{\text {loss }}^{*}=S_{\text {gain }}^{-1}$, this relation uniquely determines the phase difference $\varphi$ between the edge mode term $t$ and resonance term in Eq. (4), and yields [26]

$$
\cos \varphi=-\frac{\left(\Gamma_{1}+\Gamma_{2}\right) t}{2 \sqrt{\Gamma_{1} \Gamma_{2}}} .
$$

The transmission spectrum reflecting the interference between a continuum state and a single discrete mode can be expressed as $|\tau|^{2}=|t|^{2} \frac{(q+\delta)^{2}}{\delta^{2}+1}$ [17], with the normalized frequency, $\delta=\left(\omega-\omega_{0}\right) / \Gamma, \omega_{0}$ is the central frequency, $\Gamma$ is the decay rate of energy in the resonant mode, and $q$ is the shape parameter determined by the coupling between resonant and nonresonant modes. $q$ is obtained from the frequency difference between the central frequency of the discrete mode and the zero intensity point in the transmission spectrum. The value of $t$ is estimated from the peak signal $T_{\max }=$ $|t|^{2}\left(q^{2}+1\right)$. The final analytical fit is shown in Figs. 3(a) and $3(\mathrm{~b})$. Without loss, the phase change would be $-\pi$ and the transmission would vanish. In the measurement, the phase change is affected by internal dissipation and by the presence of other modes. The data are fit using three cavity modes at 20.812, 20.922, and 21.046 GHz in the model. The contribution of each mode at the peak and valley of the signal is shown in Figs. 3(c) and 3(d). The edge mode and resonance mode interfere destructively at the minimum in intensity. The analytical results ignore the nearby modes, so that the phase at the two ends of the spectrum does not align with the measurement.

We consider the quasinormal mode at $20.922 \mathrm{GHz}$. It should be noticed that the parameters obtained in the fit are not uniquely determined; however, $\Gamma_{1}$ cannot be zero. Comparing Eq. (4) with the standard Fano expression for the interaction of the continuum with a single mode [17] gives $q^{2}+1=\frac{4 \Gamma_{1} \Gamma_{2}}{\Gamma_{\mathrm{tot}}^{2} t^{2}}$ [26]. Thus $\frac{\Gamma_{1}}{\Gamma_{\text {tot }}}>\frac{1-\sqrt{1-\left(q^{2}+1\right) t^{2}}}{2} \sim 0.3$. We conclude that at least $30 \%$ of the linewidth of the cavity mode is due to coupling to the backward channel $s_{1-}$. Since the forward and backward channels only support the spin-up-polarized and spin-down-polarized mode, respectively, the large value of $\frac{\Gamma_{1}}{\Gamma_{\text {tot }}}$ indicates that the cavity is coupled to the backward as well as the forward edge channels and pseudo-spin-polarization is not conserved for the electromagnetic interaction with the cavity. Based on the Hamiltonian approximation mentioned previously, the disorder introduced here is proportional to $\widehat{s_{z}}$, so that this kind of disorder should not mix the spin-up and spin-down states. We might expect spin flipping to be inhibited when the disorder has TR symmetry. However, in the experiment, the Dirac cone is at $21 \mathrm{GHz}$ and a band gap of $\sim 1 \mathrm{GHz}$ is opened. This band gap is not a small perturbation, and we cannot determine the extent to which this approximation is valid. Our measurements show this Hamiltonian approximation does not work well over the entire band gap in the presence of a large resonant cavity. Spin coupling is not negligible.

The reflection of an edge mode is demonstrated in COMSOL simulations. The intensity distribution in the metacrystal at

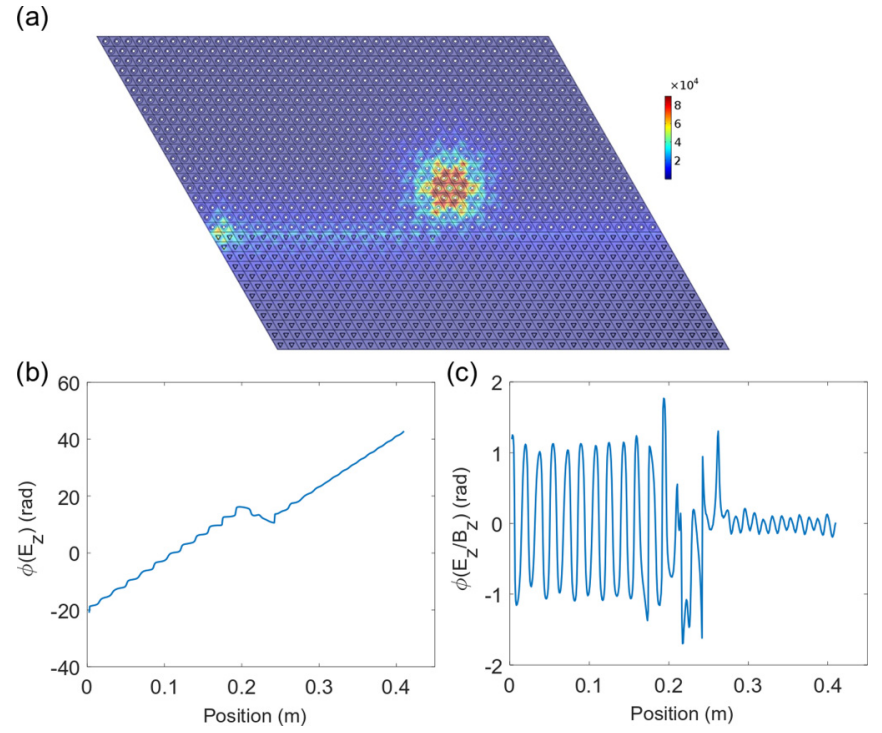

FIG. 4. Reflection induced by cavity. (a) Simulations of the intensity distribution of the wave at $21.02 \mathrm{GHz}$. The region of cavity is between 0.2 and $0.25 \mathrm{~m}$. (b) The phase change along the boundary. (c) The phase difference between $E_{z}$ and $B_{z}$ along the boundary.

21.02 $\mathrm{GHz}$, which is on resonance with the fifth mode, is shown in Fig. 4(a). The intensity is stronger in front of the cavity than behind as a result of the reflection of the wave propagating along the domain wall toward the cavity. To further demonstrate the reflection of the wave, we plot the phase of $E_{z}$ along the boundary line in Fig. 4(b). The cavity lies at a distance of between 0.2 and $0.25 \mathrm{~m}$ from the input. For $x>0.25 \mathrm{~m}$, the phase change increases linearly, indicating a forward propagating wave. In contrast, the wave in front of the cavity is modulated with a period of one half the wavelength as a result of the interference of the incident and reflected waves.

A simulation of the phase difference between $E_{z}$ and $B_{z}$ along the boundary is plotted in Fig. 4(c). The forward (backward) edge mode corresponds to spin-up (spin-down) states, which are the bonding (antibonding) combinations of the TE and TM modes. The phase difference between $E_{z}$ and $B_{z}$ for the forward and backward edge mode is $0 / \pi$. The phase difference is seen in Fig. 4(c) to oscillate between $-\pi / 3$ and $\pi / 3$ before the cavity due to interference. After the cavity, the phase difference between $E_{z}$ and $B_{z}$ is 0 , corresponding to a forward propagating mode.

The delay time in transmission at a given frequency is the delay of a pulse in the limit of diverging pulse length and vanishing bandwidth. It is equal to the derivative of the phase of the transmitted field with angular frequency $d \phi / d \omega$. The negative phase derivative at the zero-intensity point is an indication of the reshaping of the pulse at this frequency [27].

\section{GENERALIZATION TO SYSTEM WITHOUT TR SYMMETRY}

The analysis of the transmission spectrum shows that reflection is present in this TI system. However, transmission along the edge can be robust in a nonreciprocal system, as it 
(a)

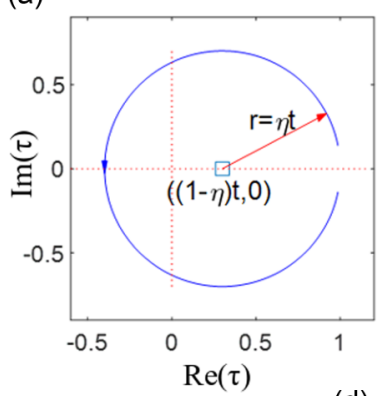

(b)

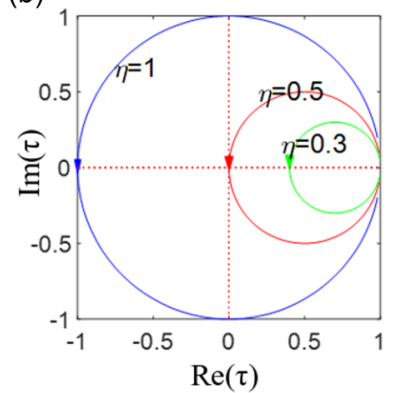

(c)

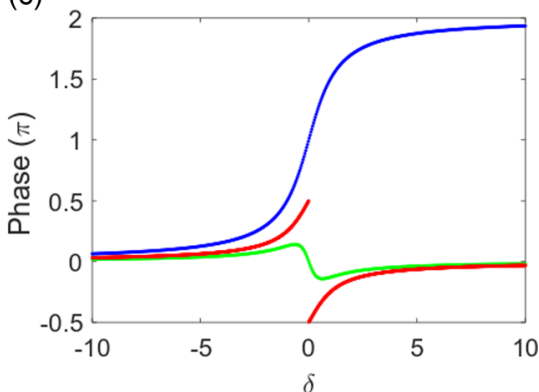

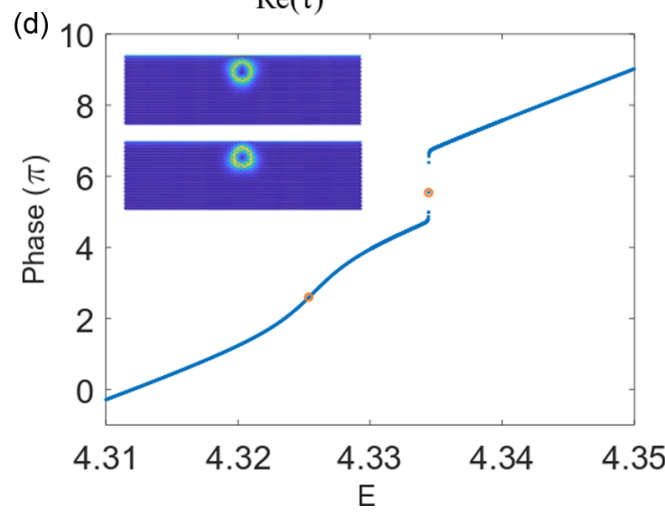

FIG. 5. Transmission through a nonreciprocal system. (a) General complex representation of transmission. (b) Complex representation for three cases with $\eta=1,0.5$, and 0.3 with phase variation as shown in (c). The arrow represents the direction of increasing $\delta$. (d) Tightbinding simulation following the Haldane model $H=\sum_{i} c_{i}^{\dagger} \lambda_{i} c_{i}+\sum_{\langle i j\rangle} c_{i}^{\dagger} t c_{j}+\sum_{\langle\langle i j\rangle\rangle} c_{i}^{\dagger} i \lambda_{\mathrm{SO}} v_{i j} c_{j}$, for on-site energy $\lambda_{i}=4$, nearest-neighbor hopping $t=-1$, and spin-obit coupling strength $\lambda_{\text {So }}=0.1$. Inside the cavity, $\lambda_{\text {SO }}=-0.1 . v_{i j}= \pm 1$. The blue line is the phase change of the transmitted field. Two discrete modes are excited. The inset shows the intensity distribution at the two energies indicated by the red dots.

is for the chiral edge state in a gyromagnetic photonic crystal [28-30]. We now consider the Fano resonance in a sample which only supports the forward channel.

In the case of a single discrete mode, the matrix $D$ in Eq. (1) becomes the outgoing coupling coefficient $d$ while $K$ becomes the incoming coupling coefficient $k$. Mann et al. [31] demonstrate that $t d^{*}=-k$ for a nonreciprocal system. Equation (2) can be rewritten to give [32]

$$
\begin{aligned}
\tau & =t+\frac{d k}{i\left(\omega_{0}-\omega\right)+\Gamma_{\mathrm{tot}}} \\
& =t\left[1-\frac{2 \Gamma_{\mathrm{rad}}}{i\left(\omega_{0}-\omega\right)+\Gamma_{\mathrm{tot}}}\right] \\
& =t\left(1-\frac{2 \eta}{1-i \delta}\right),
\end{aligned}
$$

where $\Gamma_{\text {rad }}=\frac{d d^{*}}{2}$ is the linewidth due to outgoing coupling, $\eta=\frac{\Gamma_{\text {rad }}}{\Gamma_{\text {tot }}}$, and $\delta=\left(\omega-\omega_{0}\right) / \Gamma_{\text {tot }}$.

The complex representation of $\tau$ is a circle centered at $[(1-\eta) t, 0]$ with radius $\eta t$, as shown in Fig. 5(a). As shown in Figs. 5(b) and 5(c), the circle crosses the origin when $\eta=0.5$, and there is an abrupt phase change of $-\pi$. For $\eta>0.5$, the phase increases by $2 \pi$ through the mode. In contrast, the phase derivative will be negative for $\eta<0.5$ as shown in the green curve in Fig. 5(c).

We further check this in the tight-binding model. The effect of a cavity was emulated in the lossless system $(\eta=$ 1) following the Haldane model [33]. Numerical results are obtained using the open-source package KWANT [34]. The transmission coefficient is unity since there is no backward propagating channel. We therefore focus on the phase change. The phase variation of the transmitted field is shown in Fig. 5(d). The phase increases by $2 \pi$ twice, once gradually between 4.322 and 4.33 and once sharply near 4.334 , indicating that the cavity supports two modes within this frequency range. The speckle patterns at the central frequencies of these two modes are plotted in the inset. In this case, we find the phase derivative equals the integral of the intensity over the sample for unit incident flux from the left divided by $2 \pi$, so that density of states (DOS) is propotional to the intensity integral.

\section{CONCLUSION}

We have observed a Fano resonance between a continuum edge mode and an extended defect in a time-reversal-invariant TI metacrystal. In contrast to previous work, in which the edge state resisted spin flipping in the presence of point defects, the defect flips a spin and the wave can be backscattered $[8,20]$. The transmission time near resonance increases at a rate reflecting the energy inside the system. In the present work, we find backscattering induced by an extended cavity, which accounts for $30 \%$ of the linewidth of the cavity mode. Thus, it is not possible to increase the transport time while maintaining perfect transmission in a bianisotropic structure with TR symmetry by introducing a cavity. This design can be used as a narrow-band filter for the transmitted signal along the edge in the TI whose complex frequency can be adjusted by the structure of the cavity, the dissipation rate, 
and the distance to the edge. The same cavity design can be utilized in systems without reciprocity to produce narrow peaks in delay time without backscattering. Based on the coupled-mode theory, the phase change is positive as long as the decay rate due to internal dissipation is smaller than the coupling rate between the edge and cavity. Transmitted pulses can be modified and the time delay lengthened by introducing a cavity in a nonreciprocal system. This work points the way toward a broader class of disordered TI systems in which the edge mode is coupled to extended defects along the length of the edge. The defects may be extended cavities arranged either periodically or randomly. Defect modes may also arise in systems with random disorder in which spatially localized modes are introduced into the band gap as a result of Anderson localization.

\section{ACKNOWLEDGMENTS}

This work is supported by the National Science Foundation (Grant No. NSF/DMR/-BSF: 1609218) and the succeeding grant. We thank Xiang Ni and Alexander B. Khanikaev for designing the bianisotropic structure.
[1] L. Lu, J. D. Joannopoulos, and M. Soljačić, Topological photonics, Nat. Photonics 8, 821 (2014).

[2] A. B. Khanikaev and G. Shvets, Two-dimensional topological photonics, Nat. Photonics 11, 763 (2017).

[3] M. Z. Hasan and C. L. Kane, Topological insulators, Rev. Mod. Phys. 82, 3045 (2010).

[4] M. Hafezi, E. A. Demler, M. D. Lukin, and J. M. Taylor, Robust optical delay lines with topological protection, Nat. Phys. 7, 907 (2011).

[5] M. Hafezi, S. Mittal, J. Fan, A. Migdall, and J. M. Taylor, Imaging topological edge states in silicon photonics, Nat. Photonics 7, 1001 (2013).

[6] S. Mittal, J. Fan, S. Faez, A. Migdall, J. M. Taylor, and M. Hafezi, Topologically Robust Transport of Photons in a Synthetic Gauge Field, Phys. Rev. Lett. 113, 087403 (2014).

[7] T. Ma, A. B. Khanikaev, S. H. Mousavi, and G. Shvets, Guiding Electromagnetic Waves around Sharp Corners: Topologically Protected Photonic Transport in Metawaveguides, Phys. Rev. Lett. 114, 127401 (2015)

[8] X. Cheng, C. Jouvaud, X. Ni, S. H. Mousavi, A. Z. Genack, and A. B. Khanikaev, Robust reconfigurable electromagnetic pathways within a photonic topological insulator, Nat. Mater. 15, 542 (2016).

[9] Y. Kang, X. Ni, X. Cheng, A. B. Khanikaev, and A. Z. Genack, Pseudo-spin-valley coupled edge states in a photonic topological insulator, Nat. Commun. 9, 1 (2018).

[10] T. Baba, Slow light in photonic crystals, Nat. Photonics 2, 465 (2008).

[11] B. Bahari, A. Ndao, F. Vallini, A. El Amili, Y. Fainman, and B. Kanté, Nonreciprocal lasing in topological cavities of arbitrary geometries, Science 358, 636 (2017).

[12] S. Barik, A. Karasahin, S. Mittal, E. Waks, and M. Hafezi, Chiral quantum optics using a topological resonator, arXiv:1906.11263v1 [physics.optics].

[13] M. J. Mehrabad, A. P. Foster, R. Dost, E. Clarke, P. K. Patil, I. Farrer, J. Heffernan, M. S. Skolnick, and L. R. Wilson, A semiconductor topological photonic ring resonator, Appl. Phys. Lett. 116, 061102 (2020).

[14] J. Guglielmon and M. C. Rechtsman, Broadband Topological Slow Light through Higher Momentum-Space Winding, Phys. Rev. Lett. 122, 153904 (2019).

[15] M. Xiao and S. Fan, Photonic Chern insulator through homogenization of an array of particles, Phys. Rev. B 96, 100202(R) (2017).
[16] Z.-Y. Ong and C. H. Lee, Transport and localization in a topological phononic lattice with correlated disorder, Phys. Rev. B 94, 134203 (2016).

[17] U. Fano, Effects of configuration interaction on intensities and phase shifts, Phys. Rev. 124, 1866 (1961).

[18] S. Fan, Sharp asymmetric line shapes in side-coupled waveguide-cavity systems, Appl. Phys. Lett. 80, 908 (2002).

[19] K. Lai, T. Ma, X. Bo, S. Anlage, and G. Shvets, Experimental realization of a reflections-free compact delay line based on a photonic topological insulator, Sci. Rep. 6, 1 (2016).

[20] A. B. Khanikaev, S. Hossein Mousavi, W.-K. Tse, M. Kargarian, A. H. MacDonald, and G. Shvets, Photonic topological insulators, Nat. Mater. 12, 233 (2013).

[21] C. L. Kane and E. J. Mele, Quantum Spin Hall Effect in Graphene, Phys. Rev. Lett. 95, 226801 (2005).

[22] Y. Xu, Y. Li, R. K. Lee, and A. Yariv, Scattering-theory analysis of waveguide-resonator coupling, Phys. Rev. E 62, 7389 (2000).

[23] S. Li, Y. Wang, R. Jiao, L. Wang, G. Duan, and L. Yu, Fano resonances based on multimode and degenerate mode interference in plasmonic resonator system, Opt. Express 25, 3525 (2017).

[24] W. Suh, Z. Wang, and S. Fan, Temporal coupled-mode theory and the presence of non-orthogonal modes in lossless multimode cavities, IEEE J. Quantum Electron. 40, 1511 (2004).

[25] J. Wiersig and J. Main, Fractal Weyl law for chaotic microcavities: Fresnel's laws imply multifractal scattering, Phys. Rev. E 77, 036205 (2008)

[26] J. W. Yoon, M. J. Jung, S. H. Song, and R. Magnusson, Analytic theory of the resonance properties of metallic nanoslit arrays, IEEE J. Quantum Electron. 48, 852 (2012).

[27] P. Sebbah, O. Legrand, and A. Z. Genack, Fluctuations in photon local delay time and their relation to phase spectra in random media, Phys. Rev. E 59, 2406 (1999).

[28] S. Raghu and F. D. M. Haldane, Analogs of quantum-Halleffect edge states in photonic crystals, Phys. Rev. A 78, 033834 (2008).

[29] Z. Wang, Y. D. Chong, J. D. Joannopoulos, and M. Soljačić, Reflection-Free One-Way Edge Modes in a Gyromagnetic Photonic Crystal, Phys. Rev. Lett. 100, 013905 (2008).

[30] C. He, X.-C. Sun, X.-P. Liu, M.-H. Lu, Y. Chen, L. Feng, and Y.-F. Chen, Photonic topological insulator with broken time-reversal symmetry, Proc. Natl. Acad. Sci. USA 113, 4924 (2016).

[31] S. A. Mann, D. L. Sounas, and A. Alù, Nonreciprocal cavities and the time-bandwidth limit, Optica 6, 104 (2019). 
[32] S. A. Mann, D. L. Sounas, and A. Alù, Broadband delay lines and nonreciprocal resonances in unidirectional waveguides, Phys. Rev. B 100, 020303(R) (2019).

[33] F. D. M. Haldane, Model for a Quantum Hall Effect without Landau Levels: Condensed-Matter Realiza- tion of the Parity Anomaly, Phys. Rev. Lett. 61, 2015 (1988).

[34] C. W. Groth, M. Wimmer, A. R. Akhmerov, and X. Waintal, KWANT: A software package for quantum transport, New J. Phys. 16, 063065 (2014). 